\title{
Avaliação de qualidade no seguimento de hipertensos
}

VANDA PALÁCIOS, JOSÉ NUNES

\section{RESUMO}

Objectivo: Avaliar a qualidade dos registos efectuados nos processos clínicos de doentes hipertensos do Centro de Saúde de Sintra (CSS).

Dimensão: Competência técnico-profissional.

Unidade de Estudo: Profissionais - 8 clínicos Gerais que voluntariamente aderiram ao programa. Doentes - 20 hipertensos por cada clínico geral. Total de histórias dínicas avaliadas - 160 (20x8), seleccionadas aleatoriamente.

Tipo de Avaliação: Interna.

Tipo de Dados: Processo.

Fontes de Dados: Para identificaç̃o dos casos: fichas Idade/Sexo. Para revisão - História Clínica. Relação Temporal Avaliação/Acção Avaliada - Avaliação retrospectiva.

Critérios: 20 critérios explicitos e normativos, definidos consensualmente pelos Clínicos Gerais participantes.

Medidas Correctoras Introduzidas: Educativas e estruturais.

Palavras-chave:

Avaliação de qualidade, Hipertensão arterial, Registos.

damente, procedimentos inadequados e/ou insuficientes, estrutura inadaptada, reduzida acessibilidade, etc.)

Reconhecendo estas dificuldades no controlo de doentes hipertensos, um grupo de Clínicos Gerais (CG) do CSS decidiu desenvolver um Ciclo de Garantia de Qualidade no seguimento destes doentes, tendo este trabalho como objectivo apresentar os resultados desta primeira avaliação.

\section{OBEctivos}

\section{INTRODUÇ̃̃O}

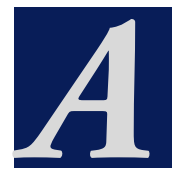

hipertensão arterial (HTA) é um importante factor de risco de morbo-mortalidade de cérebro-cárdio-vascular. ${ }^{1,2,3}$ Pela sua elevada incidência e prevalência, constitui um importante problema de Saúde Pública. ${ }^{4}$

Vários estudos têm demonstrado que a prevenção e controlo adequados da HTA conduzem a uma diminuição da incidência de acidentes vasculares cere-

Vanda Palácios Interna do Internato Complementar de Clinica Geral

José Nunes Assistente de Clínica Geral Centro de Saúde de Sintra brais, enfartes do miocárdio, dissecções aórticas e claudicações intermitentes. ${ }^{5}$ Contudo, nem sempre o controlo tensional é tarefa fácil, quer por dificuldades de adesão terapêutica do doente, quer por deficiente desempenho técnico-profissional dos Serviços de Saúde (nomea-
Pretende-se avaliar a qualidade dos registos efectuados nos processos clínicos de doentes hipertensos do CSS. ${ }^{6,7,8,9}$

\section{Métodos}

\section{Dimensão do estudo}

Analisou-se a competência técnico-profissional dos médicos participantes.

\section{Unidade de estudo}

Voluntariamente, 8 CG predispuseram-se a participar neste estudo. Foram seleccionados aleatoriamente 20 processos clínicos de doentes hipertensos por cada CG participante. A amostra perfez um total de 160 processos clínicos individuais avaliados.

A aplicação dos critérios foi efectua- 
da relativamente ao ano anterior à data da avaliação. Tratou-se de um estudo transversal.

\section{Fontes de dados}

Utilizaram-se as fichas Idade/Sexo para identificação dos hipertensos. Aos processos aleatoriamente seleccionados, foi feito o estudo dos registos efectuados nos mesmos.

\section{Critérios}

Após o conhecimento dos CG aderentes ao projecto em questão, efectuou-se uma reunião a fim de serem definidos os critérios a aplicar. ${ }^{1,2,4,10}$ Neste encontro, foi elaborada uma lista consensual de 20 critérios explícitos e normativos, que no entender dos intervenientes deveriam ser cumpridos para que se pudesse considerar adequado o seguimento dos doentes hipertensos (Guadro I). Estes foram especificados de forma clara e inequívoca, fazendo-se acompanhar de esclarecimentos, quando oportunos, bem como das excepções admissiveis.

Para cada médico, foi calculado o respectivo Indice de Suficiência (IS), ${ }^{11}$ definindo-se este como sendo:

IS $=\frac{\text { total de critérios cumpridos }}{\text { Total de critérios }} \times 100$

\section{Tipo de avaliação}

A avaliação foi de tipo interno, aplicada a $8 \mathrm{CG}$, realizada pela Autora, na presença do Médico de Família de cada um dos doentes em questão. A cada um $\operatorname{dos} 8$, foi atribuído um número aleatório, tendo apenas deste conhecimento o próprio e a Autora. A dita avaliação processou-se pois em regime de semi-anonimato, evitando ferir susceptibilidades, ou provocar as inevitáveis e sempre desagradáveis comparações entre colegas, até porque num ciclo de garantia de qualidade o termo de comparação de cada elemento é ele próprio.

\section{Tipo de dados}

Foi avaliado o processo, ou seja, os dados analisados foram os procedimentos registados, de acordo com os critérios previamente definidos.

\section{REsUltados}

No Guadro II, encontra-se um resumo dos resultados obtidos em percentagem. Por exemplo, em relação ao médico $n .{ }^{\circ} 1$, temos que em 16 das suas 20 histórias clínicas o critério hábitos tabágicos se encontrou satisfeito, isto é, a existência ou não de hábitos tabágicos encontrava-se registada no Processo Individual de Saúde desses 16 doentes. Quanto ao critério pulsos periféricos, verificou-se que, e ainda em relação ao médico em questão, apenas 15\% das histórias clínicas apresentavam satisfeito esse critério.

Já em relação ao registo de medição de tensão arterial, notou-se que em 95\% das histórias clínicas, este critério foi cumprido. Verificou-se em relação a outro médico a existência de um erro técnico: o registo de classificação de HTA mediante a repercussão nos órgãos-alvos em 100\% das histórias clínicas avaliadas, conquanto apenas $50 \%$ dos doentes tivessem efectuado fundoscopia.

Quanto ao IS (última linha do Quadro II), registou-se o facto deste ter sido bastante variável de médico para médico, oscilando entre 30 e $73 \%$ (Figura 1).

Pela observação da Figura 2, pode-se verificar que, a par de critérios que de uma forma geral foram bem satisfeitos (medição da tensão arterial, parâmetros analíticos - uremia, glicemia em jejum e colesterolemia, registo dos fármacos hipotensores prescritos, registo dos antecedentes pessoais e familiares, hábitos alcoólicos e tabágicos), outros 


\section{QUADRO I}

\section{CRITÉRIOS}

\section{DEFINIÇÃO}

1. Ant. Pessocis

2. Ant. Familiares

3. Háb. Tabágicos

4. Háb. Alcoólicos

5. Altura

6. Peso

7. Tens. Arterial

8. Ausc. Cardíaca

9. Pulsos Perifér.

10. E.C.G.

11. Glicemia em Jejum

12. Uremia

13. Creatininemia

14. Ionograma

15. Colestrol Total

16. Fundoscopia

17. Repercussão nos Órgãos-Alvo

18. Consulta nos últimos 2 meses

19. Procedimentos

20. Fármacos registo (em qualquer parte da hist. clínica) da existência ou não de AVC, Diabetes, HTA

registo (em qualquer parte da hist. clínica) da existência ou não de AVC, Diabetes, HTA

registo em qualquer local da história clínica

registo em qualquer local da história clínica

registada, pelo menos I vez em qualquer parte da hist. clínica

registado, pelo menos 1 vez, no último ano

registada, pelo menos 1 vez, no último ano

registada, pelo menos 1 vez, no último ano

registado o resultado da palpação, pelo menos 1 vez, no último ano

registado, pelo menos 1 vez, no último ano

registada, pelo menos 1 vez, no último ano

registada, pelo menos 1 vez, no último ano

registada, pelo menos 1 vez, no último ano

registado, pelo menos 1 vez, no último ano

registado, pelo menos 1 vez, nos últimos 5 anos

registado o resultado, pelo menos 1 vez, no último ano

registo em qualquer local da história clínica

consulta registada

mencionado em qualquer ponto da hist. clínica ter recebido, no último ano, qualquer procedimento educativo higieno-dietético

registo da terapêutica hipotensora em qualquer parte da hist. clínica
ALTERNATIVA

acamado ou retido no domićlio

amputado

se não tiver medicação hipotensora

não considerar, se $>65$ anos

considerar os últimos 6 meses se controlada (T.A. diast. <95) ou ter sido convocado e não ter respondido

controlo da HTA só com medidas higieno-dietéticas 


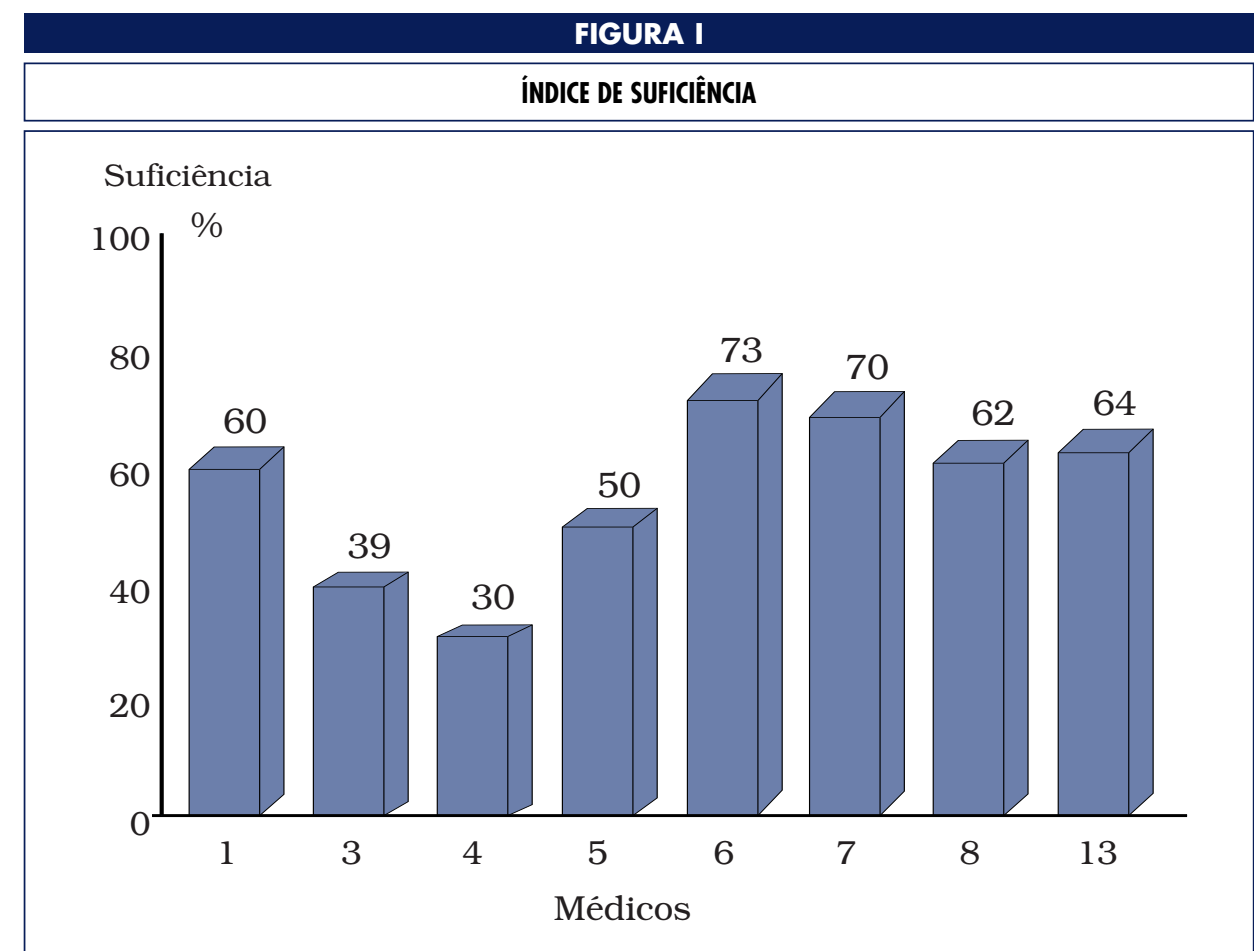

FIGURA II

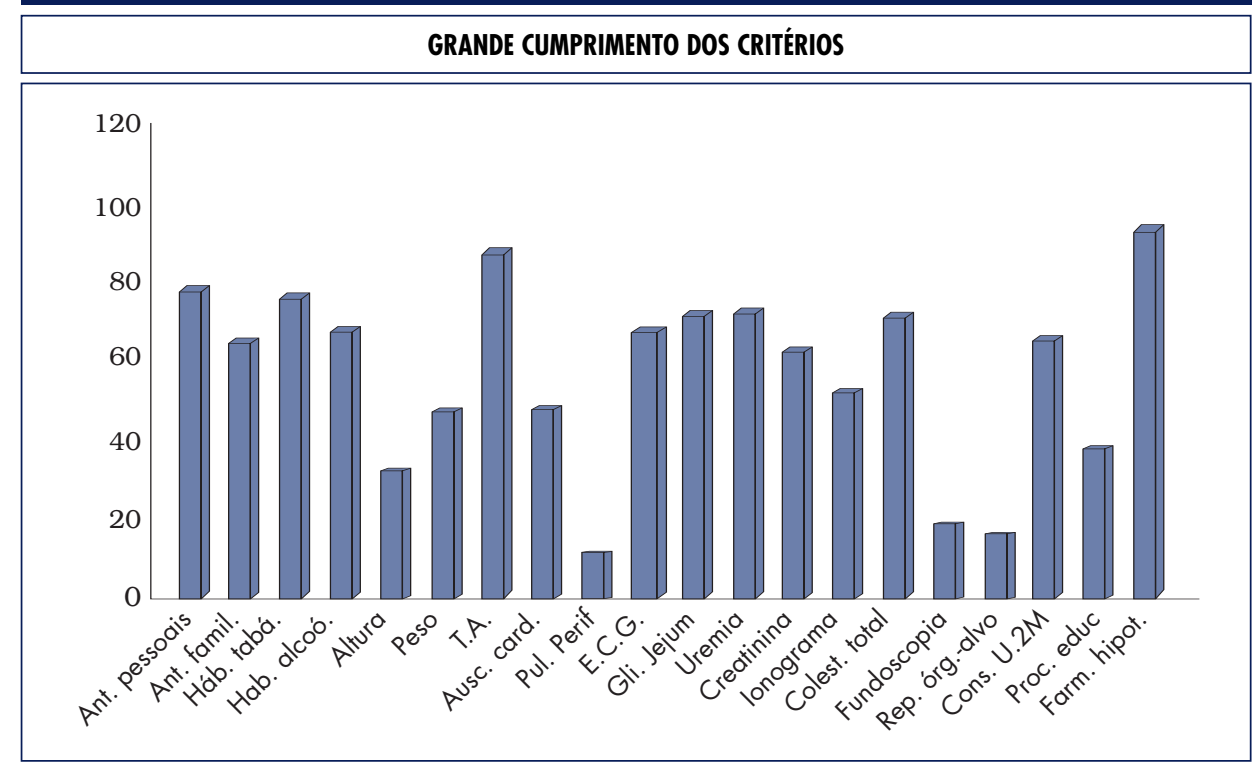




\begin{tabular}{|c|c|c|c|c|c|c|c|c|}
\hline \multicolumn{9}{|c|}{ QUADRO IV } \\
\hline \multicolumn{9}{|c|}{ RESUMO DOS RESULTADOS (\%) } \\
\hline $\begin{array}{l}\text { Médico № } \\
\text { Critérios }\end{array}$ & 1 & 3 & 4 & 5 & 6 & 7 & 8 & 13 \\
\hline Ant. Pessoais & 50 & 70 & 75 & 90 & 100 & 100 & 90 & 55 \\
\hline Ant. Familiares & 35 & 50 & 65 & 75 & 100 & 70 & 65 & 60 \\
\hline Háb. Tabágicos & 80 & 60 & 20 & 70 & 100 & 95 & 90 & 90 \\
\hline Háb. Alcoólicos & 75 & 15 & 15 & 65 & 100 & 95 & 90 & 90 \\
\hline Altura & 55 & 0 & 0 & 30 & 85 & 20 & 10 & 55 \\
\hline Peso & 90 & 20 & 25 & 50 & 80 & 25 & 20 & 65 \\
\hline T. A. & 95 & 85 & 75 & 75 & 100 & 90 & 100 & 85 \\
\hline Ausc. Cardíaca & 50 & 30 & 0 & 65 & 15 & 60 & 80 & 80 \\
\hline Pulsos Perifér. & 15 & 0 & 0 & 10 & 15 & 5 & 5 & 30 \\
\hline E.C.G. & 80 & 25 & 45 & 45 & 100 & 85 & 80 & 85 \\
\hline Glicemia em Jejum & 85 & 55 & 45 & 55 & 95 & 90 & 90 & 80 \\
\hline Uremia & 85 & 55 & 40 & 50 & 90 & 90 & 90 & 80 \\
\hline Creatininemia & 70 & 30 & 15 & 55 & 70 & 95 & 85 & 80 \\
\hline lonograma & 70 & 55 & 10 & 35 & 55 & 85 & 50 & 60 \\
\hline Colesterolemia Total & 75 & 45 & 65 & 55 & 100 & 85 & 80 & 70 \\
\hline Fundoscopia & 10 & 0 & 5 & 35 & 20 & 50 & 5 & 15 \\
\hline Reperc. Órgãos-Alvo & 5 & 0 & 0 & 5 & 10 & 100 & 0 & 0 \\
\hline Consulta nos últimos $2 \mathrm{M}$. & 90 & 70 & 0 & 50 & 90 & 60 & 85 & 80 \\
\hline Proc. educacionais & 30 & 25 & 10 & 10 & 100 & 70 & 30 & 30 \\
\hline Fárm. hipotensores & 95 & 95 & 90 & 85 & 95 & 100 & 95 & 95 \\
\hline Índice de Suficiência & 60 & 39 & 30 & 50 & 73 & 70 & 62 & 64 \\
\hline
\end{tabular}

houve com um baixo grau de cumprimento (medição de altura, classificação da HTA em graus mediante a repercussão nos órgãos-alvos, palpação dos pulsos periféricos, fundoscopia). Foi assim, muito variável o grau de cumprimento dos diferentes critérios definidos.

\section{Discussão e Conclusões}

Em nosso entender, a identificação de critérios foi um dos momentos mais rendiveis em termos formativos, na medida em que, para além de ter motivado uma pesquisa bibliográfica cuidadosa, e de ter proporcionado uma in- teressante discussão interpares acerca de um tema de indiscutivel importância - a hipertensão arterial, implicou por si só, e desde logo, a correcção de alguns procedimentos.

Um aspecto interessante foi o facto das reacções dos CG participantes em face dos resultados obtidos terem sido muito díspares. Para alguns, estes foram absolutamente ao encontro das suas previsões; para outros, os resultados constituíram verdadeiras surpresas (num e noutro sentido). Foi contudo unânime a decisão de melhorar drasticamente o cumprimento de alguns critérios, nomeadamente os que neste trabalho de avaliação de quali- 
dade se revelaram com menores indices de satisfação.

Foi sugerida a criação de uma Folha-Tipo, destinada à solicitação de observação oftalmológica, folha essa a ser utilizada pelos colegas que se considerassem menos à vontade na realização desse exame. Outra reacção em resposta aos resultados proveio de alguns dos elementos intervenientes, que sentiram necessidade de reorganizar de um modo mais funcional a sua consulta de doentes hipertensos.

$\mathrm{Na}$ sequência desta avaliação de qualidade, foi pois sentida a necessidade de introdução de medidas correctoras de natureza estrutural e educativa. Sabendo que num ciclo de garantia de qualidade as fases de avaliação e de introdução de medidas correctoras se sucedem, no sentido de alcançar o melhor nivel de qualidade possivel, far-se-á uma nova avaliação, se possivel, dentro de 12 meses, com o intuito de avaliar o impacto das correcções introduzidas.

Como meta mais ambiciosa, pretende-se o desenvolvimento de trabalhos de avaliação de qualidade aplicados a outros problemas consideravelmente prevalecentes em Clínica Geral.

\section{REFERÊNCIAS BIBLIOGRÁFICAS}

1. García MDF, Wichman MD, Ferré MLF, Navarro CL, Solas MT. Hipertensão Arterial. In: Zurro AM, Perez JFC. Manual de Cuidados Primários. Lisboa: Farmapress Edições Lda., 1991: 287-303

2. Williams GH, Braunwald E. Doença Vascular Hipertensiva. In: Braunwald E et al. Harrison - Medicina Interna. Rio de Janeiro: Editora Guanabara Koogan S.A., 1998; 1: 95163.

3. S.A Hipertensão Arterial. Notícias Médicas - Ed. O Médico Prático, 1995; 24 (2274): 5-7

4. Dias JL, Imperatori E, Miguel JP, Mota
JCB, Nunes AC, Sakelarides C. Hipertensão Arterial e Cuidados de Saúde Primários. Lisboa: Direcção Geral dos Cuidados de Saúde Primários, 1984: 11-107

5. Swales JD. Hipertensão. Update, 1994; 6 (65): 44-8

6. Vivancos RV, Ferrer JT. Implementación del protocolo de hipertensión en un área básica de salud como base para una auditoria médica. Aten. Prim., 1994; 13 (6): 320-2.

7. Ordoño JM, Diez A, Esquisabel R, Medrano MT, Ayesa G, Pereda L et al. Introdución del control de calidad en un centro de salud: evaluación del registro de la historia del usuario y de los problemas detectados. Atend. Prim., 1993; 12 (3): 156-9.

8. Delgado E, Hernández E, Izquierdo JM, Landa V, Lejarza R, López Palacios V et al Evaluación del grado de cumplimiento del protocolo de hipertensión arterial. Atend. Prim., 1990; 7 (10): 635-40.

9. Puime AO, Hernández PJS. Avaliação e Controlo de Qualidade em Cuidados de Saúde Primários. In: Zurro AM, Perez JFC. Manual de Cuidados Primários. Lisboa: Farmapress Edições Lda, 1991: 241-53.

10. Carr AA. Hipertensão: um problema que não é só da pressão arterial. Post. Grad. Med. - Edição Port., 1995; 3 (5): 22-9.

11. Vuori HV. El control de calidad en los Servicios Sanitarios - Conceptos y Metodologia. Ed. Masson, S.A., 1991.

\section{Agradecimentos}

Aos colegas participantes:
Ana Margarida Levy
Fernando Bolina
Helena Fonseca
Josefina Marau
Marina Belo
Olímpia Abreu
Susana Santos 


\section{Estudos OrigINAIS}

\section{QUALITY ASSESSMENT IN THE FOLLLOW-UP OF PACIENTS WITH HYPERTENSION}

\section{SUMMARY:}

Objective: To access the quality of clinical record of pacients with hypertension in the Health Centre of Sintra.

Dimension: Technical and professional skills.

Unit of Study: Health professionals. Eight General Practitioners agreed to participate.

Patients: One hundred and sixty clinical records were randomly selected, corresponding to 20 records per G.P.

Type of Assessment: Internal.

Source of Data: Records age/sex for identification of cases. Clinical data for revision. Retrospective assessment.

Criteria: Twenty explicit and normative criteria, defined by participating GPs in consensus meeting. Corrective Measures: Structural and educational.

Key words:

Quality Assessment, Arterial Hypertension, Clinical Records.

Recebido em 21/08/95

Aceite para publicação em 24/06/99

Endereço para correspondência

Vanda Palácios

Rua Dr. José António Serrano

Lt 99 - $6^{\circ}$ Frente

Massamá

2745 Queluz 